\title{
Atendimento prestado pelos Serviços de saúde à Mulher Vítima de Violência Sexual
}

\author{
Eugênia Silva Santos ${ }^{l}$; Maria Antonieta Pereira Tigre Almeida ${ }^{2}$
}

\begin{abstract}
Resumo: A violência sexual consiste em um ato hostil e agressivo empregado pelos ofensores como forma de degradar, humilhar, aterrorizar e dominar. Faz parte da história, atravessa os séculos e é observada em diferentes sociedades e culturas, classes sociais, raças e etnias. As consequências são graves e a mulher é exposta a diferentes riscos que comprometem sua saúde, podendo provocar distúrbios psicológicos como também afeta aspectos de ordem física como lesões de pele, infecções sexuais, lacerações, doenças sexualmente transmissíveis e até mesmo a gravidez. Os profissionais e serviços de saúde apresentam grande importância na relação com a mulher vítima de violência sexual, tanto no atendimento decorrentes da violência como também na assistência imediata. $O$ presente estudo teve por objetivo elaborar uma revisão sistemática sobre os conhecimentos disponíveis na literatura acerca do atendimento prestado pelos serviços de saúde à mulher vítima de violência sexual. Para fomentar a sua construção realizou-se buscas em artigos de revistas indexadas em acervos eletrônicos a partir de palavras-chaves. A assistência à mulher em situação de violência sexual deve ser organizada através de conhecimento científicos atualizados, bases epidemiológicas sustentáveis e tecnologia apropriada. A implementação de protocolos assistenciais e de ações de educação continuada para os profissionais da saúde que atuam com a assistência a mulher vítima de violência sexual poderão contribuir em estratégias de atendimento voltadas para o cuidado e bem-estar destas. O atendimento pautado nos direitos, acolhimento, humanização e integralidade da assistência deve ser priorizado nos serviços de saúde à mulher.
\end{abstract}

Palavras-Chave: agressão sexual, atenção primária a saúde, saúde da mulher, violência

\section{Assistance provided by the Health Services to Women Victims of Sexual Violence}

\begin{abstract}
Sexual violence consists of a hostile and aggressive act employed by the offenders as a way of degrading, humiliating, terrorizing and dominating. It is part of history, goes through the centuries and is observed in different societies and cultures, social classes, races and ethnicities. The consequences are serious and the woman is exposed to different risks that compromise her health and can cause psychological disorders as well as affect physical aspects such as skin lesions, sexual infections, lacerations, sexually transmitted diseases and even pregnancy. Health professionals and services have great importance in the relationship with women victims of sexual violence, both in the care due to violence and in the immediate assistance. The present study had the objective of elaborating a systematic review on the available knowledge in the literature about the health services provided to women victims of sexual violence. In order to promote its construction, searches were carried out on journal articles indexed in electronic collections from keywords. Assistance to women in situations of sexual violence should be organized through up-to-date scientific knowledge, sustainable epidemiological bases and appropriate technology. The implementation of care protocols and continuing education actions for health professionals who work with the assistance of women victims of sexual violence may contribute to care strategies focused on their care and well-being. The care based on the rights, reception, humanization and integrality of care should be prioritized in the health services to women.
\end{abstract}

Keywords: sexual agression, primary health care, women's health, violence

\footnotetext{
${ }^{1}$ Aluna do Curso de Enfermagem da Faculdade Independente do Nordeste - FAINOR, Vitória da Conquista - BA, 2017. E-mail: ogenamodesto@gmail.com

${ }^{2}$ Docente da Faculdade Independente do Nordeste - FAINOR, Vitória da Conquista - BA.
} 


\section{Introdução}

A violência contra a mulher pode se manifestar de várias formas e com diferentes graus de severidade, estas formas de violência não se produzem isoladamente, mas fazem parte de uma sequência crescente de acontecimentos.

Um tipo de violência comum é a violência sexual que vem sendo um sério problema de saúde pública aparecendo como uma das principais causas de morbidade e mortalidade feminina tanto no Brasil como no mundo (SOUZA e OLIVEIRA, 2016). Consiste em uma grave violação dos direitos humanos que pode ocasionar em danos irreversíveis à saúde mental e física da vítima, além de gerar custos sociais (OSHIKATA et al., 2011).

A violência sexual apresenta associação com as definições dos papéis sociais femininos e masculinos, ou seja, com o estudo de gênero. O papel social do homem passou a ser definido, tanto culturalmente como historicamente, como autoridade, os valores e prerrogativas culturais que definem o papel sexual masculino tradicional são o poder, a dominação, a força, a violência e a superioridade, já os valores e prerrogativas culturais que definem o papel sexual feminino são a submissão, a passividade, a fraqueza e a inferioridade (SOUZA, 2008).

De acordo com a Organização Mundial de Saúde (OMS, 2002) a violência sexual incide em todo ato sexual não desejado, ou ações de comercialização e/ou utilização da sexualidade de uma pessoa mediante qualquer tipo de coerção. É um ato que desconhece barreiras culturais, classes sociais, níveis socioeconômicos e limitações individuais, podendo ocorrer em espaços privado ou público, e atingir mulheres de todas as faixas etárias. Quanto ao agressor, pode ser cometida por indivíduos de todas as classes sociais, e, na maioria das vezes apresenta relações de afeto ou laços consanguíneos com a vítima, como pais, padrastos, namorados, maridos, amigos, entre outros.

As consequências são graves e a mulher é exposta a diferentes riscos que comprometem sua saúde. Assim, a violência sexual é capaz de provocar distúrbios psicológicos provenientes do trauma, como também afeta aspectos de ordem física, como lesões de pele, infecções sexuais, lacerações, doenças sexualmente transmissíveis, AIDS e a gravidez (DINIZ et al., 2007). As vítimas presentam também alta predisposição para alguns sintomas psiquiátricos, 
como depressão, pânico, somatização, tentativa de suicídio, abuso e dependência de substâncias psicoativas (AZEVEDO et al., 2013).

O Ministério da Saúde, no ano de 1999, criou a Norma Técnica de Prevenção e Tratamento dos Agravos Resultantes da Violência Sexual contra Mulheres e Crianças, que favoreceu recursos técnicos aos municípios para que estes elaborassem uma rede de atendimento às vítimas de violência sexual (BRASIL, 2012). Os serviços de atenção as mulheres em situação de violência sexual, chamados serviços de porta de entrada e de referência para o acompanhamento, são essenciais para melhorar a qualidade de vida das pessoas que enfrentam esse trauma.

Nos últimos anos houve um expressivo aumento de mulheres em situação de violência sexual urbana que recorrem aos serviços de saúde, estes, oferecem além do suporte ambulatorial atenção na área de saúde mental.

Os serviços de saúde consistem em um sistema de atenção orientado para a manutenção, restauração e promoção da saúde, visando o bem-estar físico e psicológico das pessoas. É importante ressaltar que os serviços de saúde não contemplam somente o diagnóstico e tratamento de doenças ou transtornos, mas abrangem também a prevenção e difusão de aspectos que favorecem uma vida saudável.

Dessa forma, os profissionais e serviços de saúde apresentam grande importância na relação com a mulher vítima de violência sexual, tanto no atendimento decorrentes da violência como também na assistência imediata. $\mathrm{O}$ atendimento e assistência adequada além de proporcionar um apoio para a vítima é importante para evitar complicações futuras e um desgaste maior.

A relevância social e acadêmica em abordar esse tema, está em compreender os aspectos que envolvem esse problema de saúde pública buscando estratégias para minimizá-los já que os prejuízos não são direcionados apenas a mulher, mas a sociedade em geral, uma vez que ela está inserida em um contexto de relações sociais e todos os envolvidos sofrem com as repercussões dessa violência.

Diante disso, o presente estudo teve por objetivo elaborar uma revisão sistemática sobre os conhecimentos disponíveis na literatura acerca do atendimento prestado pelos serviços de saúde à mulher vítima de violência sexual. 


\section{Metodologia}

O estudo foi realizado por meio de uma revisão de literatura. Para fomentar a sua construção realizou-se buscas em artigos de revistas indexadas em acervos eletrônicos como Scientific Eleronic Library Online (Scielo) e google acadêmico a partir de palavras-chaves.

Para a pesquisa foram selecionados artigos, livros, monografias, dissertações e teses publicadas entre os anos de 2002 e 2016, além de uma análise exploratória para o reconhecimento dos artigos que interessavam para o estudo de maneira geral.

A pesquisa bibliográfica consiste em explicar e mostrar um problema, utilizando certos conhecimentos disponíveis a partir da temática em estudo. Incide ainda em revelar o pesquisador o conhecimento disponível na área, identificando as teorias produzidas, analisando-as e avaliando sua contribuição para auxiliar a compreender ou explicar o problema tendo a análise da investigação do texto (KOCHE, 1997).

\section{Resultados e Discussão}

\section{Os tipos de violência e seus impactos na vida da mulher}

De modo geral, a violência pode ser definida como o uso de palavras ou de ações que maltratam as pessoas, e também o uso abusivo ou injusto do poder ou da força resultando em lesões, sofrimento, tortura ou até mesmo a morte.

De acordo com Presser (2007) a violência contra a mulher não significa resultado de ocorrências inexplicáveis ou de desvios patológicos ou de condutas, mas sim em práticas desenvolvidas na organização social alicerçada na desigualdade, em que o poder é exercido pelos que se acham no direito de intimidar e controlar.

Nesse sentido, o autor ainda destaca o uso do conceito de violência de gênero, reconhecida como qualquer ato que resulta ou pode resultar em danos ou sofrimentos físico, 
sexual, psicológico ou patrimonial a mulher, inclusive ameaças, coerção ou privação de liberdade, castigos, maus tratos, pornografia e agressão sexual.

Menegheli e Hirakata (2011) ressalta que as violências baseadas em gênero compreendem agressões de caráter físico, psicológico, sexual e patrimonial.

Segundo Marinheiro, Vieira e Souza (2006) a violência praticada contra as mulheres é denominada de violência de gênero por correlacionar com a condição de subordinação da mulher na sociedade, e englobam a agressão física, sexual, psicológica e econômica.

Guedes e Fonseca (2011) também cita que a naturalização da violência contra a mulher é constituída a partir da desigualdade entre os sexos, que vem historicamente sendo gerada como mecanismos ideológicos que permitem a sujeição das mulheres nas relações de gênero, partindo dos conceitos de inferioridade.

Atualmente, a violência contra as mulheres tem sido um dos temas mais relevantes no âmbito da Saúde Pública e dos direitos humanos, justamente devido aos prejuízos à saúde e ao desenvolvimento psicossocial dessas mulheres (GARCIA-MORENO et al., 2006).

Jorge (2006) enfatiza que do ponto de vista econômico, a violência representa um custo bastante elevado devido aos gastos dos serviços de saúde com o tratamento das lesões e traumatismos provenientes dela.

Diante disso, no intuito de proteger a mulher da violência e aumentar o rigor das punições sobre os agressores, em 22 de setembro de 2006, entrou em vigor a Lei Maria da Penha, que em seu art. $1^{\circ}$ define como seu objetivo coibir e prevenir a violência doméstica e familiar contra a mulher.

Quanto aos tipos de violência contra a mulher, Cavalcanti (2006) dividiu para melhor compreensão de cada categoria, são elas:

- Violência física: consiste em atos de acometimento físico sobre o corpo da mulher através de tapas, chutes, golpes, queimaduras, mordeduras, estrangulamentos, punhaladas, mutilação genital, tortura, assassinato, etc.;

- Violência psicológica: é a ação ou omissão destinada a degradar ou controlar as ações, comportamentos, crenças e decisões de outra pessoa por meio de intimidação, manipulação, ameaça direta ou indireta, humilhações, isolamento ou qualquer outra conduta que implique prejuízo à saúde psicológica, a autodeterminação ou ao desenvolvimento pessoal; 
- Violência moral: consiste no assédio moral, em que o patrão ou chefe agride física ou psicologicamente sua funcionária com palavras, gestos ou ações, bem como na prática de crimes de calúnia, injúria e difamação contra a mulher;

- Violência patrimonial: é aquela praticada contra o patrimônio da mulher, muito comum em casos de violência doméstica e familiar;

- Violência institucional: é a praticada nas instituições prestadoras de serviços públicos, como hospitais, postos de saúde, escolas, delegacias, no sistema prisional, etc.;

- Violência de gênero ou de raça: é aquela praticada em razão do preconceito, discriminação e exclusão social;

- Violência doméstica e familiar: é a ação ou omissão que ocorre no espaço de convívio permanente de pessoas, com ou sem vínculo familiar, inclusive as esporadicamente agregadas. É aquela praticada por membros de uma mesma família, aqui entendida como a comunidade formada por indivíduos que são ou se consideram aparentados, unidos por laços sanguíneos (naturais), por afinidade ou vontade expressa.

- Violência sexual: identifica- se com qualquer atividade sexual não consentida, incluindo também o assédio sexual. Sua ocorrência é bastante comum durante os conflitos armados, bem como em razão do tráfico internacional de mulheres e crianças para fins sexuais ou pornográficos;

Para Basile e Smith (2011) dentre os vários tipos de violência contra a mulher, a violência sexual consiste naquela que pode causar consequências mais impactantes que as demais. Além disso, é um fenômeno de conceituação complexa pela sua interface com os aspectos sociais, culturais, religiosos e econômicos (BRASIL, 2011).

Em estudo realizado por Marinheiro, Vieira e Souza (2006) com o objetivo de determinar a prevalência dos tipos de violência entre 265 mulheres, de 18 a 49 anos, usuárias de um centro de saúde em Ribeirão Preto-SP, constataram que a violência psicológica ocorreu pelo menos uma vez na vida de $41,5 \%$ delas, a violência física em $26,4 \%$ e a violência sexual em $9,8 \%$.

Outra pesquisa de Schraiber et al. (2002) realizada também no município de São Paulo com usuárias de uma unidade básica de saúde, com idade entre 15 e 49 anos, verificaram que $44,4 \%$ delas relataram ocorrência de episódio de violência física e 11,5\% de violência sexual. 
Ao analisar 2502 mulheres na faixa etária de 15 anos ou mais, Schraiber et al. (2007) observaram que $43 \%$ da população do estudo relatou ter sido vítima de violência praticada por um homem. Destas, um terço relatou ter sofrido violência física, $13 \%$ violência sexual e $27 \%$ traumas psicológicos.

A violência sexual faz parte da história e atravessa os séculos, sendo observada em diferentes sociedades e culturas, classes sociais, raças e etnias. Na Idade Média era uma prática associada aos rituais de masculinidade e só era condenada pelos penitenciais da Igreja quando envolvia rapto, pois esta condição a convertia em crime contra a propriedade (VIGARELLO, 1998). Oliveira (2007) também cita as guerras, em que as mulheres eram violentadas como símbolo de conquista e barbárie que circundava esse tipo de ação.

Para Franco (2015) a violência sexual consiste em um ato hostil e agressivo empregado pelos ofensores como forma de degradar, humilhar, aterrorizar e dominar. Na visão de Oliveira (2007) é um ato não decorrente do desejo sexual ou amoroso, mas sim de uma demonstração extrema de poder do homem sobre as mulheres, visando transforma-las em objeto do seu poder e de sua autonomia.

Na definição da Organização Mundial de Saúde (OMS) é um ato sexual ou tentativa de obtê-lo sem o consentimento da mulher por meio de atitudes coercitivas e intimatórias como força física, graves ameaças, uso de armas e pressão psicológica (OSHIKATA et al., 2011).

Em um conceito mais amplo, a Lei Maria da Penha, em seu Art.7, considera a violência sexual contra a mulher como

\footnotetext{
III - a violência sexual, entendida como qualquer conduta que a constranja a presenciar, manter ou a participar de relação sexual não desejada, mediante intimidação, ameaça, coação ou uso da força; que a induza a comercializar ou a utilizar, de qualquer modo, a sua sexualidade, que a impeça de usar qualquer método contraceptivo ou que a force ao matrimônio, à gravidez, ao aborto ou à prostituição, mediante coação, chantagem, suborno ou manipulação; ou que limite ou anule o exercício de seus direitos sexuais e reprodutivos. (BRASIL, 2006, p.17).
}

É um grave problema que além de violar os direitos humanos causam grandes impactos físicos, psíquicos e sociais, e, em 1993 passou a ser recohecida como um problema de saúde pública global pela Organização Pan-Americana da Saúde (OPAS) e OMS (KRUG et al., 2002). A partir daí várias ações foram elaboradas para incentivar estudos sobre violência contra a 
mulher e propor intervenções para a prevenção, atendimento adequado, humanizado e normatizado (BRASIL, 2012).

A luta histórica e constante dos movimentos de mulheres e outros componentes da sociedade civil, ao denunciar a arbitrariedade dos padrões de relacionamentos baseados em modelos masculinos, desencadeou na consolidação de recursos jurídicos e sociais de proteção à mulher em situação de violência (BRASIL, 2012).

Atento a essa questão e sensível às reivindicações dos movimentos feministas e de mulheres, o Ministério da Saúde, nos últimos anos, tem assumido papel de destaque quanto ao enfretamento à violência, de modo intra e intersetorial por meio de ações articuladas com a Secretaria Especial de Políticas para as Mulheres no âmbito do Pacto Nacional de Enfretamento à Violência Sexual (BRASIL, 2012).

\section{Os serviços de sáude na atenção à mulher vítima de violência sexual}

A ação dos seviços de sáude exerce papel de destaque na vida das pessoas, em especial das mulheres em situação de violência, e também consiste em uma garantia dos seus direitos humanos, considerando que grande parte da população tem contato com o sistema de saúde em algum momento, mesmo que seja por outra razão que não seja a agressão (BRASIL, 2012).

Conforme Sagot (2002) apesar da existência de serviços públicos e ONGs direcionadas a atenção às mulheres em situação de violência, nota-se que muitas não encontram o amparo que almejam e alternativas para superação do trauma, enfrentando assim uma trajetória marcada por dificuldade, desestímulo, inexistência ou falta de acesso às instituições e serviços. Em países de Terceiro Mundo, as mulheres em situação de violência e deprimidas costumam ser medicadas e quase sempre tratadas com sintomáticos como analgésicos, polivitamínicos e medicamentos para insônia (OMS, 2004).

Para Auengraum (2002) estudos apontam que o uso mais frequente dos serviços de saúde, ambulatoriais e hospitales são nos casos de violência doméstica. Já Krug et al. (2002) diz que a procura pelo serviço de saúde ocorre pela necessidade de cuidado provocada pela violência, pelas sequelas psicológicas, além de outros sintomas que podem aparecer. 
Várias medidas tem sido implantadas para disponibilizar atendimento efetivo às mulheres que sofreram violência sexual, o Ministério da Saúde elaborou, desde 1999, a Norma Técnica "Prevenção e Tratamento dos Agravos Resultantes da Violência Sexual contra Mulheres e Adolescentes", que apresenta um protocolo de atendimento para as mulheres que sofreram violência sexual (BRASIL, 2011).

De acordo com a referida Norma Técnica, a assistência à mulher em situação de violência sexual deve ser organizada através de conhecimento científicos atualizados, bases epidemiológicas sustentáveis e tecnologia apropriada. As ações de atenção à saúde devem está disponíveis para toda a população, do município ou região, incubindo às instituições assegurar cada etapa do atendimento, abrangendo as medidas de emergência, o acompanhamento, reabilitação e tratamento dos eventuais impactos da violência sexual sobre a saúde física e mental da mulher (BRASIL, 2012).

Diante desses aspectos, constata-se que a violência sexual consiste em um importante agravo à saúde e violação dos direitos das mulheres e que exige atenção, em especial dos serviços de saúde (GARCIA et al., 2008).

De acordo com Lettiere, Nakano e Rodrigues (2008) os serviços de saúde são referências e porta de entrada das vítimas de violência pois, o contato com elas contribuem para reconhecer e acolher essas mulheres.

Bedone e Faúndes (2010) destacam quem em 2010 existiam no Brasil 442 serviços de saúde para atendimento de violência sexual e 60 serviços especializados para atendimento de procedimentos relacionados à violência sexual previstos em lei. Contudo, um aspecto a se observar é em relação a qualidade do atendimento, essencial para proporcionar segurança e conforto a quem recorra a esses serviços.

Durante a assistência, as mulheres vítimas da violência sexual devem ser informadas, sempre que possível, sobre tudo que será realizado em cada fase do atendimento e a importância de cada uma delas, sua vontade deve ser respeitada aceitando possível recusa de algum procedimento, e deve ser oferecido atendimento psicológico e medidas de fortalecimento para auxiliá-las a enfrentar os conflitos e problemas decorrentes da situação vivida (BRASIL, 2012).

A atenção à saúde da mulher em situação de violência deve superar o modelo biomédico e buscar um atendimento que proporcione o seu bem-estar. A equipe de saúde deve apresentar 
habilidade, começando no acolhimento e na orientação das vítimas, o acolhimento consiste no tratamento digno e respeitoso desde a escuta, reconhecimento e aceitação de diferenças, principalmente com resolubilidade e acesso a assistência, tendo a saúde como um direito universal e um dever do Estado (BRASIL, 2011).

A tarefa de acolher deve ser uma prática holística de equipe multiprofissional envolvida no amparo das vítimas de violência sexual e deve estar presente de forma transversal durante todo o período de contato com essa mulher (BRASIL, 2011). O autor acrescenta ainda que profissionais sensibilizados e capacitados devem promover a escuta privilegiada, evitado julgamentos, preconceitos e comentários, respeitando a autonomia das mulheres mantendo uma correlação de confiança.

Além desse suporte, o ideal é que os serviços de saúde ofereçam um atendimento pautado na Norma Técnica preconizada pelo Ministério da Saúde. Andalaf et al. (2012) ao pesquisar uma amostra representativa de municípios brasileiros, observaram que 1.395 serviços de saúde prestavam atendimento a mulheres e crianças que sofreram violência sexual, contudo, somente $8 \%$ apresentavam um protocolo de atendimento em conformidade com a referida norma.

No entanto, para enfrentar a violência sexual, e de gênero como um todo, não basta normatizar procedimento, é fundamental discutir sobre o tema afim de consolidar conhecimento com marcos políticos nacionais e internacionais vigentes e com as estratégias e as ações de enfretamento à violência contra as mulheres que são aplicadas no país, ou seja, o processo de acolhimento e orientação profissional deve ser livre de julgamentos ou valores morais (BRASIL, 2012).

Faúndes et al. (2006) em seu estudo sobre os procedimentos indicados e seus resultados no atendimento de urgência em mulheres vítimas de violência sexual, salientam que as vítimas esperam muito mais que a simples aplicação de protocolos, anseiam receber um atendimento digno, respeitoso e acolhedor cabendo aos profissionais o reconhecimento de seu importante papel com exercício ético e responsável de medidas protetoras de sua saúde e de seus direitos humanos.

Ainda segundo Faúndes et al. (2006), a equipe de saúde deve entender que imediatamente depois de uma violência sexual, a mulher necessita de apoio emocional, pois sua 
autoestima e seu ego podem ter sido mais afetados que uma lesão física que venha a apresentar. Além disso, é preciso perceber que para quem sofreu tal crime, o simples fato de procurar o sistema de saúde e/ou delegacia de polícia já é um agravo decorrente da violência (BRASIL, 2012).

Na visão de Franco (2015) o atendimento às mulheres que sofreram violência sexual vai além da oferta de condutas e procedimentos, requer cumplicidade do profissional, acolhimento, escuta e empenho. Durante o atendimento é essencial que as pessoas demonstrem simpatia e compreensão, disposição a ouvi-la com respeito e interesse bem como esteja preparada para prestar apoio emocional. Contudo, nem todo profissional pode estar apto, em termos emocionais ou até mesmo capacitados de forma técnica para atender essas mulheres (OLIVEIRA, 2007).

Um princípio fundamental, conforme evidenciam Faúndes et al. (2006) é que o atendimento não deve se limitar à emergência, pois a violência sexual apresenta consequências a longo prazo que precisam ser prevenidas e tratadas tão logo apareçam. Os autores afirmam ainda que devido a multiplicidade dos impactos, o atendimento à vítima de violência sexual exige a atuação de uma equipe multidisciplinar, além disso, muitas vezes o atendimento psicológico e social pode se estender a alguns membros da família.

O ideal é que o atendimento seja concedido pela equipe multidisciplinar, cuja composição pode diversificar conforme os recursos disponíveis nas unidades de saúde. Conforme a Norma Técnica "Prevenção e Tratamento dos Agravos Resultantes da Violência Sexual contra Mulheres e Adolescentes" é desejável que seja composta por médicos, enfermeiros e assistentes sociais, embora a falta de um dos profissionais, exceto o médico, não torna o atendimento inviável, o importante é que sejam sensíveis, capacitados e aptos a perceber esse problema como um fenômeno social que pode causar muitos agravos à saúde da mulher. Contudo, serviços de saúde de referência para os casos de maior complexidade podem adicionar à equipe multidisciplinar especialistas em infectologia, cirurgia, traumatologia, psiquiatria, entre outras (BRASIL, 2012).

Para Facuri et al. (2013) é imprescindível que os serviços de saúde atendam as vítimas de forma ágil, acolhedora, em bom ambiente capaz de atuar nas preocupações imediatas (lesão física, doenças sexualmente transmissíveis, gravidez) e também nas dificuldades psíquicas. 
Segundo Franco (2015) as situações relevantes no tratamento da violência sexual nos serviços de saúde é o risco de gravidez, as DST's e o HIV.

É importante que o serviço de saúde realize exames físicos completo, exame ginecológico, coleta de amostra para diagnóstico de infecções genitais e coleta de material para identificação do provável autor da agressão, além do preenchimento da "ficha de notificação e investigação de violência doméstica, sexual e/o outras violências (BRASIL, 2012).

Cavalcanti, Flach e Farias (2012) apontam que o acesso das mulheres em situação de violência sexual nas unidades de saúde pode ocorrer de forma espontânea ou através do encaminhamento por outros serviços, como Instituto de Medicina Legal, Delegacia Especializada de Atendimento à Mulher, Centro de Referência a Mulher, entre outros. Os autores realçam ainda que é fundamental que os profissionais de saúde em contato com a vítima mantenham uma postura cuidadosa, de forma a estabelecer um bom vínculo, em que a sensibilidade ao problema é mais importante que compartilhar a dor da paciente, que deve se sentir acreditada e acolhida.

O contato inicial com o serviço de saúde quase sempre é realizado por enfermeiros que fazem os encaminhamentos de acordo a gravidade da violência, sendo as ocorrências classificadas segundo o intervalo de tempo entre a agressão e o contato com o serviço de saúde, podendo ser imediatas, até cinco dias após a violência sexual e tardias, após cinco dias da agressão (FACURI et al., 2013). Os autores observam que o contato precoce com o serviço de saúde é primordial para favorecer a qualidade de atendimento quanto a introdução de profilaxias para DST's e anticoncepção de emergência, contribuindo assim para amenizar o sofrimento da vítima e os impactos decorrentes da violência.

Outra preocupação expressa pelas políticas públicas em relação à violência contra as mulheres é a falta de registro dos casos pelos serviços de saúde, que poderiam contribuir com o planejamento e execução de medidas para prevenir as ocorrências de violência e agilizar o atendimento das vítimas (SOUZA et al., 2015).

Como forma de diminuir essa falta e favorecer a efetivação de estratégias de prevenção, o Governo Federal estabeleceu a exigência de notificação compulsória em casos de violência contra a mulher por meio da Lei $\mathrm{n}^{\circ} 10778$, de 24 de novembro de 2003, em que todos os casos de violência contra a mulher, seja física, sexual ou psicológica, que forem atendidos em serviços 
públicos ou privados precisam ser notificados através de preenchimento de uma ficha (BRASIL, 2003).

Nesse mesmo sentido, com a finalidade de avaliar a proporção de serviços de saúde que preenchem a notificação compulsória e quais os principais obstáculos para o preenchimento do documento, Souza et al. (2015) realizaram um estudo com 172 serviços que referiram prestar atendimento às mulheres no estado de São Paulo, em foram entrevistados os profissionais do estabelecimento. De acordo com os resultados, verificou-se que a proporção de serviços que referiram sempre preencher a ficha de notificação em casos de violência sexual foi de 79,1\%, mais da metade $(53,5 \%)$ referiram ter dificuldades em relação as mulheres atendidas, um terço foi motivos direcionados a ficha e $29,7 \%$ dificuldades quanto aos profissionais.

Os autores do estudo acima mencionado concluíram que mesmo que a maior parte dos serviços de saúde referiram preenchimento da ficha de notificação compulsória, foram relatadas dificuldades para executá-la, principalmente devido à sobrecarga de trabalho dos profissionais e a incompreensão sobre a importância da notificação para a atenção integral às mulheres vítimas de violência sexual. Ainda ressaltaram a necessidade de métodos para a melhoria da formação continuada dos profissionais a fim de que a ficha de notificação compulsória seja incorporada ao atendimento das mulheres valorizando a atenção dentro das atribuições dos serviços de saúde.

A notificação tem um papel estratégico no desencadeamento de ações de prevenção e proteção, além disso é essencial nas ações de vigilância e monitoramento da situação de saúde relacionada às violências (BRASIL, 2012).

Além dos aspectos abordados, a ficha de notificação compulsória consiste ainda em um dos mecanismos das políticas públicas e um instrumento de garantia de direitos e proteção social para as mulheres vítimas de violência sexual.

\section{Considerações Finais}

A realização deste estudo possibilitou reconhecer a necessidade de rever a preparação dos profissionais de saúde que atuam no atendimento de mulheres em situação de violência

d on Line Rev. Psic. V.11, N. 35. Maio/2017 - ISSN 1981-1179 Edição eletrônica em http://idonline.emnuvens.com.br/id 
sexual afim de que possam lidar com a violência como um problema de saúde pública de fato, e que o atendimento seja realizado de forma acolhedora com atenção e cuidados que favoreçam a construção de vínculos que excedam o atendimento emergencial.

Por ser a violência um fenômeno complexo e multifacetado, abarcando vários aspectos como segurança, justiça, educação e saúde, para o seu enfrentamento são necessárias ações interdisciplinares entre diferentes campos de conhecimento e instituições sociais. Assim, as ações devem ser construídas e consolidadas através de implementação e conjugação de esforços e medidas articuladas e direcionadas a prevenção.

A implementação de protocolos assistenciais e de ações de educação continuada para os profissionais da saúde que atuam com a assistência a mulher vítima de violência sexual poderão contribuir em estratégias de atendimento voltadas para o cuidado e bem-estar destas. $\mathrm{O}$ atendimento pautado nos direitos, acolhimento, humanização e integralidade da assistência deve ser priorizado nos serviços de saúde à mulher.

\section{Referências}

ANDALAFT, J.N.; FAÚNDES, A.; OSIS, M.J.D.; PÁDUA, K.S Perfil do atendimento à violência sexual no Brasil. Femina, 40(6):301-6, 2012.

AUGENGRAUN, M.; WILSON, T.E.; ALLISTER, L. Domestic violence reported by women attending a sexually transmitted desease clinic. J Am Sex Transm Dis Assoc, 28:143-6, 2002.

AZEVEDO, Renata Cruz Soares; et al. Violência sexual: estudo descritivo sobre as vítimas e o atendimento em um serviço universitário de referência no estado de São Paulo. Cadernos de Saúde Pública, v.29, n.5, p. 889-898, 2013.

BASILE, K.C.; SMITH, S.G. Sexual violence victimization of women: prevalence, characteristics, and the role of public health. A J Lifestyle Med, 5:407-17, 2011.

BRASIL. Lei $n^{\circ}$ 11.340/2006. Lei Maria da Penha. Brasília: Secretaria Especial de Políticas para as Mulheres. 2008.

BRASIL. Ministério da Saúde. Secretaria de Atenção à Saúde. Departamento de Ações Programáticas Estratégicas. Área Técnica de Saúde da Mulher, Atenção integral para mulheres e adolescentes em situação de violência doméstica e sexual - Matriz pedagógica para formação de redes. Textos Básicos de Saúde, Brasília: Ministério da Saúde, 2011. 
BRASIL. Lei 10.778, de 24 de novembro de 2003. Estabelece a notificação compulsória, no território nacional, de caso de violência contra a mulher que for atendida em serviços de saúde públicos ou privados. Disponível em: http://www.planalto.gov.br/ccivil_03/leis/2003/110.778.htm. Acesso: 15 de março de 2017.

BRASIL. Ministério da Saúde. Secretaria de Atenção à Saúde. Departamento de Ações Programáticas Estratégicas. Prevenção e tratamento dos agravos resultantes da violência contra mulheres e adolescentes: Norma Técnica. $3^{\text {a }}$ ed. Brasília: Ministério da Saúde, 2012.

BEDONE, A.J.; FAÚNDES, A. Atendimento integral às mulheres vítimas de violência sexual: Centro de Assistência Integral à Saúde da Mulher, Universidade Estadual de Campinas. Cad Saúde Pública, 23:465-9, 2007.

CAVALCANTI, L.F.; FLACH, R.M.D.; FARIAS, R.S. Atenção às mulheres em situações de violência sexual nos serviços de saúde do Estado do Rio de Janeiro. O social em questão, ano XV, n.28, 2012.

CAVALCANTI, S.V.F. Violência doméstica: análise da Lei "Maria da Penha", n 11430/06. $3^{\mathrm{a}}$ ed. Revista Enfermagem UFPE, 7(862-9), 2006.

DINIZ, N.M.F.; ALMEIDA, L.C.G.; RIBEIRO, B.C.S.; MACÊDO, V.G. Mulheres vítimas de violência sexual: adesão à quimioprofilaxia do HIV. Revista Latino-americana de Enfermagem, 15(1), 2007.

FACURI, C.O.; FERNANDES, A.M.S.; OLIVEIRA, K.D.; ANDRADE, T.S.; AZEVEDO, R.C.S. Violência sexual: estudo descritivo sobre as vítimas e o atendimento em um serviço universitário de referência no estado de São Paulo, Brasil. Cad Saúde Pública, 29(5):889-898, 2013.

FAÚNDES, A.; ROSAS, C.F.; BEDONE, A.J.; OROZCO, L.T. Violência sexual: procedimentos indicados e seus resultados no atendimento de urgência de mulheres vítimas de estupro. Rev Bras Ginecol Obstet., 28(2):126-35, 2006.

FRANCO, Cláudia Ribeiro da Cunha. Violência sexual contra a mulher e gênero: revisão integrativa da literatura. [trabalho de conclusão de curso]. 24f. Universidade do Vale do Rio dos Sinos, Porto Alegre-RS, 2015.

GARCIA, M.V.; RIBEIRO, L.A.; JORGE, M.T.; PEREIRA, G.R.; RESENDE, A.p. Caracterização dos casos de violência contra a mulher atendidos em três serviços na cidade de Uberlândia, Minas Gerais, Brasil. Cad de Saúde Pública, 24(11):2551-2563, 2008.

GARCIA-MORENO, C.; JANSEN, H.A.; ELLSBERG, M.; HEISE, L.; WATTS, C.H. WHO Multicountry study on women's health and domestic violence against women study team, Prevalence of intimate partner violence: findings from the WHO multi-country study on women's health and domestic violence. Lancet, 368(9543):1260-9, 2006.

GUEDES, R.N.; FONSECA, R.M.G.S. A autonomia como necessidade estruturante para o enfrentamento da violência de gênero. Revista Escola de Enfermagem da USP, 45:(1731-5), 2011.

JORGE, Maria Helena Prado de Mello. Violência como problema de saúde pública. Ciência e Cultura Temas e Tendências - Violência. Revista da Sociedade Brasileira para o Progresso da Ciência, ano 54, n.1, 2002. 
KOCHE, José Carlos. Fundamentos de Metodologia Científica: teoria da ciência e prática da pesquisa. $14^{a}$ edição, Petrópolis, Editora: Vozes, 1997.

KRUG, E.G.; DAHLBERG, L.L.; MERCY, J.A.; ZWI, A.B.; LOZANO, R. World reporto n violence and health. Geneva: World Health Organization, 2002.

LETTIERE, A.; NAKANO, A.M.S.; RODRIGUES, D.T. Violência contra a mulher: a visibilidade do problema para um grupo de profissionais de saúde. Revista da Escola de Enfermagem da USP, v.42, n.2, pp.377-382, 2008.

MARINHEIRO, A.L.V.; VIEIRA, E.M.; SOUZA, L. Prevalência da violência contra a mulher usuária de serviço de saúde. Rev Saúde Pública, 40(4), 2006.

MENEGHELI, S.N.; HIRAKATA, V.N. Femicídios: homícidios femininos no Brasil. Rev Saúde Pública, v.45, n.3, p.564-574, 2011.

OLIVEIRA, E.M. Violência sexual e saúde. Cad de Saúde Pública, 23(2)455-458, 2007.

OMS. Relatório da organização Mundial da Saúde. Brasília-DF, 2002.

OSHIKATA, C.T.; BEDONE, A.J.; PAPA, M.S.F.; SANTOS, G.B.; PINHEIRO, C.D.; KALIES, A.H. Características das mulheres violentadas sexualmente e da adesão ao seguimento ambulatorial: tendências observadas ao longo dos anos em um serviço de referência em Campinas-SP. Cadernos de Saúde Pública, 27(4), 701-713, 2011.

PRESSER, Adriana Dewes. Mulheres enfrentando a violência de gênero - a voz dos operadores sociais. [dissertação]. 78f. Universidade do Vale do Rio dos Sinos, 2007.

SAGOT, M. Ruta critica de las mujeres afectadas por la violência intrafamilirar en América Latina: estudios de caso de diez paises. OPAS (Organização Pan-Americana de Saúde), 2002.

SCHRAIBER, L.B.; D’OLIVEIRA, A.F.P.L.; FRANÇA JÚNIOR, I.; PINHO, A.A. Violência contra a mulher: estudo emu ma unidade de atenção primária à saúde. Rev Saúde Pública, 36:470-7, 2002.

SCHRAIBER, L.B.; et al. Prevalência da violência contra a mulher por parceiro íntimo em regiões do Brasil. Rev Saúde Pública, 41(5)797-807, 2007.

SOUSA, M.H.; BENTO, S.F.; OSIS, M.J.D.; RIBEIRO, M.P.; FAÚNDES, A. Preenchimento da notificação compulsória em serviços de saúde que atendem mulheres que sofrem violência sexual. Rev Bras Epidemiol, 18(1):94-107, 2015.

SOUZA, Marcelly Mayara dos Santos; OLIVERA, Maconny Veríssimo Paes. Violência sexual contra a mulher e o papel do enfermeiro: revisão de literatura. [trabalho de conclusão de curso]. $21 \mathrm{f}$. Universidade Tiradentes, Aracajú-SE, 2016.

SOUZA, S.R. Comentários à lei de combate a violência contra a mulher: Lei Maria da Penha, $\mathrm{n}^{\circ}$ 11430/06. 2 ed. Curitiba: Juruá, 2008. 
Id on Line Revista Multidisciplinar e de Psicoloqia

Id on Line Multidisciplinary and Psycology Journal

VIGARELLO, Geoges. História do Estupro - Violência Sexual nos séculos XVI-XX. Rio de Janeiro: Jorge Zahar Editor, 1998.

\section{Como citar este artigo (Formato ABNT):}

SANTOA, Eugênia. S.; ALMEIDA, Maria A. P.T. Atendimento prestado pelos Serviç.os de saúde à Mulher Vítima de Violência Sexual. Id on Line Revista Multidisciplinar e de Psicologia, Abril de 2017, vol.11, n.35, p.84-99. ISSN: 1981-1179.

Recebido: 17.05.2017

Aceito: 18.05.2017 